\title{
Insectos en nidos de aves de la Argentina: Asthenes dorbignyi (Reichenbach, 1853) [Aves: Furnariidae]
}

\author{
Insects in birds' nests from Argentina: Asthenes dorbignyi \\ (Reichenbach, 1853) [Aves: Furnariidae] \\ Paola Turienzo $^{1 *} \&$ Osvaldo Di Iorio ${ }^{1}$
}

\section{RESUMEN}

Asthenes dorbignyi (Reichenbach, 1853) es un furnárido que se distribuye en los Andes de Perú, Bolivia, norte de Chile y noroeste de la Argentina entre los 2200 a los $4600 \mathrm{~m}$ sobre el nivel del mar. Los nidos son cilíndricos o globulares, verticales, con una entrada lateral cerca del techo (sin un túnel lateral), mayormente construidos en cactus columnares. Los insectos hallados en los nidos de $A$. dorbignyi en Campo Negro (Salta) y Cuesta de los Cardones (Tucumán) fueron adultos pertenecientes a Coleoptera, Hemiptera, Lepidoptera, Hymenoptera y Siphonaptera. Mientras que las especies de Coleoptera y Hemiptera utilizaron los nidos para hibernar, las Lepidoptera fueron ocasionales, utilizando el nido como refugio para la pupación, así como otros lugares disponibles, apareciendo junto con una avispa parásita Ichneumónida. Una avispa Chalcidoidea fue una especie accidental, probablemente parasitando las ootecas de las arañas utilizadas por A. dorbignyi en las camas del nido. Se discute la presencia de una especie de pulga (Siphonaptera) en los nidos de A. dorbignyi, nuevo registro de hospedador para el género Dasypsyllus. La baja diversidad de insectos en los nidos está relacionada a la baja diversidad de los biomas desérticos de altura con condiciones climáticas rigurosas.

Palabras clave: monte, hibernación, refugio, grandes alturas.

\begin{abstract}
Asthenes dorbignyi (Reichenbach, 1853) is a furnarid bird distributed in the Andean mountains of Peru, Bolivia, northern Chile and northwestern Argentina between 2200 to 4600 m upon sea level. The nests are cylindrical or globular, verticals, with a lateral entrance located near the top (without a lateral tunnel), mostly build in columnar cacti. Insects were found in nests of A. dorbignyi during june of 2007 in Campo Negro (Salta province, Argentina) and during june of 2009 in Cuesta de los Cardones (Tucumán province, from the same country). Insects obtained in the nests were adults belonging to species of Coleoptera, Hemiptera, Lepidoptera, Hymenoptera and Siphonaptera. While the Coleoptera and Hemiptera use the nests for hibernation, the Lepidoptera were occasional, using the nests as refuge for pupation as well as other suitable places, ocurring together with an ichneumonid parasite wasp. A chalcidoid wasp was other accidental species, probably parasitizing the spider cocoons used by A. dorbignyi in the beds of the nest. The presence of a flea (Siphonaptera) in the nests of A. dorbignyi is discussed, being a new host record for the genus Dasypsyllus. The low diversity of insects in these nests is correlated with the lower diversity in desertic bioma of high areas with rigurous climatic conditions.
\end{abstract}

Key words: monte, hibernation, shelter, high altitudes.

\section{Introducción}

Asthenes dorbignyi, llamado vulgarmente "canastero rojizo" en la Argentina, es un ave de 16 $\mathrm{cm}$ de longitud que se distribuye desde el sudoeste de Perú, sudoeste de Bolivia, norte de Chile hasta el oeste de la Argentina: Jujuy, Salta, Tucumán, Catamarca (Fig. 1) hasta Mendoza, variando en las distintas localidades entre los 2200 y 4600 metros de altura sobre el nivel del mar (Hoy, 1980; Vaurie, 1980). En Argentina, A. dorbignyi habita cardonales de Trichocereus a más de $2000 \mathrm{~m}$ de altura, con vegetación arbustiva y algarrobos aislados, y es la especie que se distribuye en más ambientes en el valle Calchaquí (Capllonch \& Moyano Wagner, 2010). Esta especie de ave no presenta problemas de conservación, aunque se la ha mencionado como escasa (Hoy, 1980).

\footnotetext{
1 Entomología. Departamento de Biodiversidad y Biología Experimental. Facultad de Ciencias Exactas y Naturales. $4^{\circ}$ Piso, Pabellón II, Ciudad Universitaria C1428EHA, Buenos Aires, Argentina.

* Autor por correspondencia: paolaturienzo@yahoo.com
} 


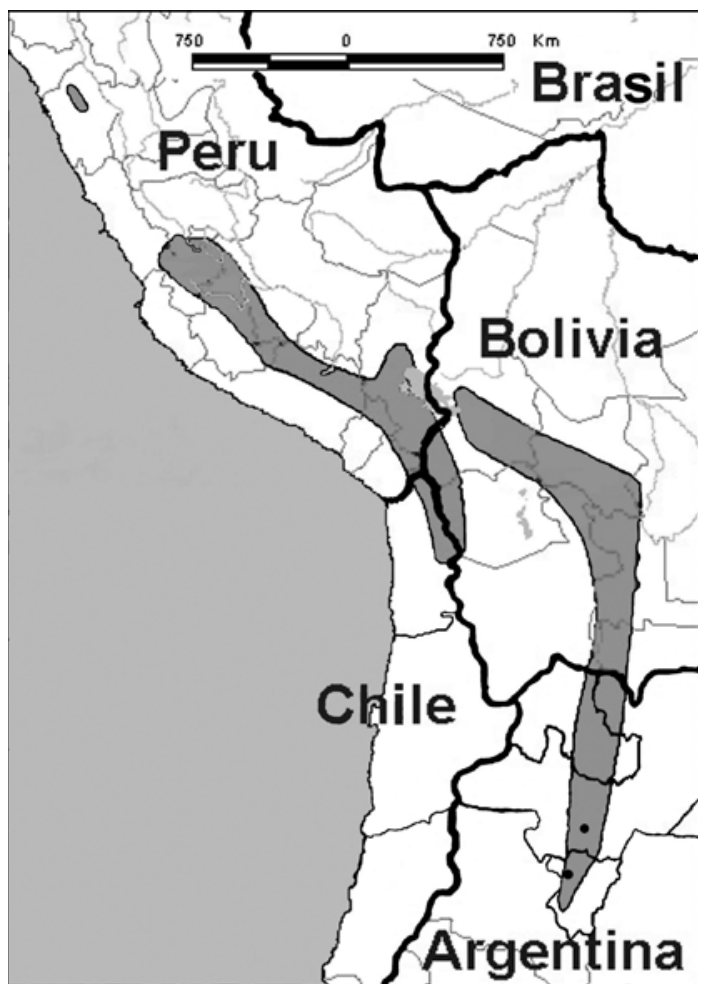

Figura 1. Distribución de Asthenes dorbignyi en Perú, Bolivia y el noroeste de Argentina (extraída de InfoNatura 2005). Los círculos negros indican las localidades en donde se muestrearon los nidos: hacia el norte, Campo Negro (Salta); al sur, Cuesta de los Cardones (Tucumán).

Los nidos de Asthenes dorbignyi fueron descriptos y mencionados en arbustos a baja altura (De la Peña, 2005), sobre cactus columnares (Narosky et al., 1983; Albanesi et al., 2009) (Figs. 2-3), en Puya raimondii (Dorst, 1957), o sobre Populus (Narosky et al., 1983). Diferencias en la construcción de los nidos en Perú y Chile respecto de los de Argentina sugieren importantes diferencias taxonómicas entre las formas arequipae y dorbignyi, las que podrían corresponder a distintas especies (Narosky et al., 1983). Los diferentes autores difieren en cuanto a que los nidos de A. dorbignyi presentan espinas (Hoy, 1980) o no (Dorst, 1957; Albanesi et al., 2009) en los materiales vegetales utilizados para su construcción.

Tanto Narosky et al. (1983) como De la Peña (2005) ilustran el nido de A. dorbignyi, pero mientras Narosky et al. (1983) lo orientan en un sentido vertical, el segundo autor lo hace en un sentido horizontal. Las observaciones realizadas en los nidos

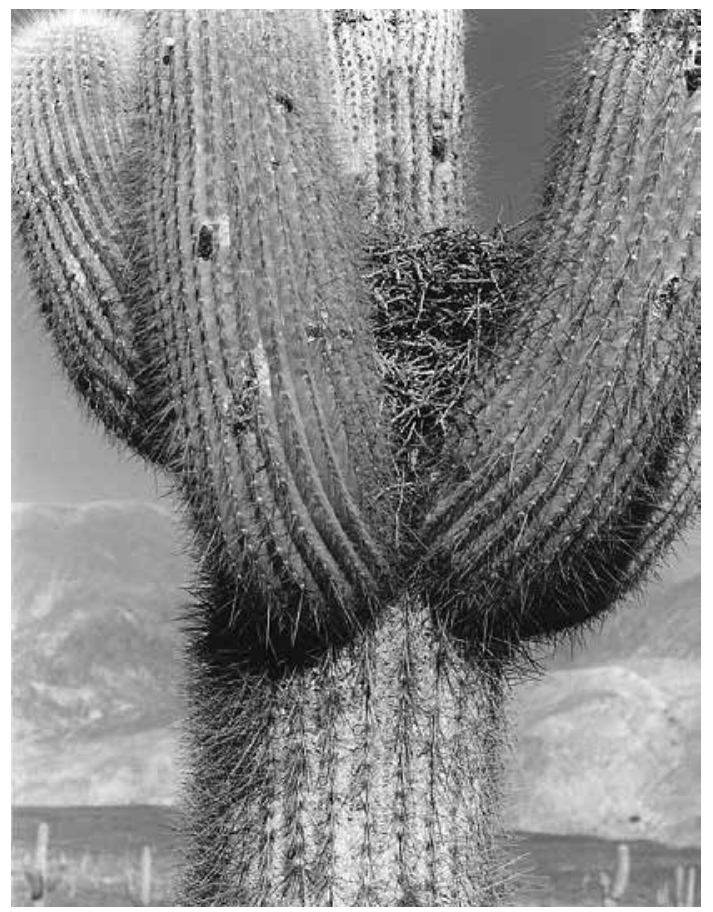

Figura 2. Nido de Asthenes dorbignyi sobre Trichocereus atacamensis en Campo Negro, provincia de Salta (Argentina).

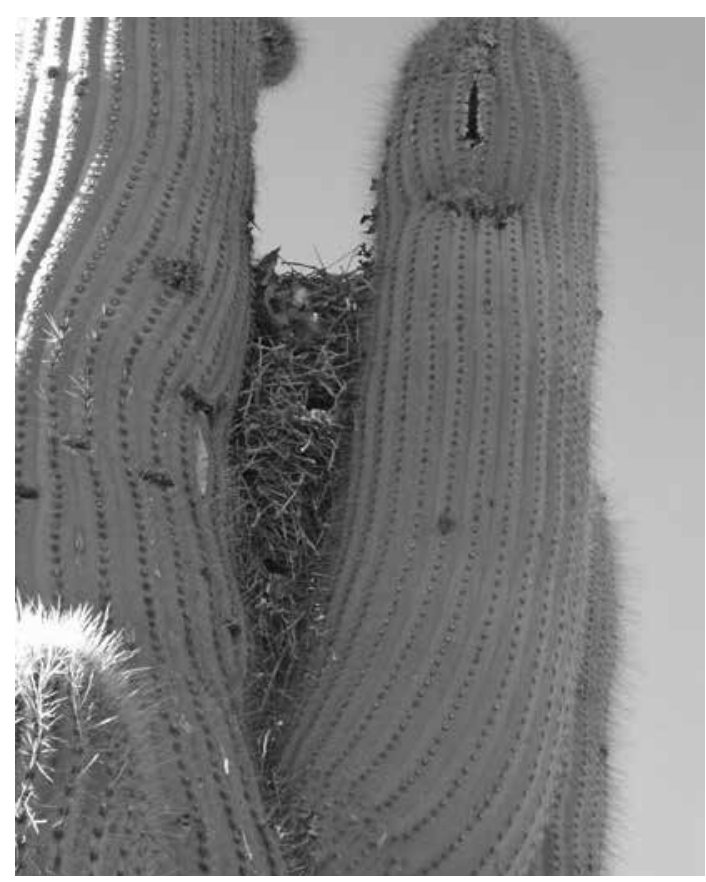

Figura 3. Nidos superpuestos de Asthenes dorbignyi sobre Trichocereus atacamensis en Cuesta de los Cardones, Tucumán (Argentina). Nótese las entradas circulares situadas lateralmente y en la parte superior de cada nido. 
de La Poma mostraron que la orientación de los nidos es en sentido vertical, como fuera establecido por Narosky et al. (1983). La entrada se ubica en el extremo superior (Fig. 3), con un conducto que lleva a la cámara de cría en el extremo inferior (Turienzo \& Di Iorio obs. pers.). Frecuentemente los nidos viejos se encuentran colapsados, es decir, aplanados, y son frecuentes los nidos superpuestos en una misma planta sostén (Fig. 3).

La cámara puede presentar lana, detritos algodonosos y pocas plumas (Narosky et al., 1983). Morrison (1939) menciona que el nido estudiado sobre cactus en Perú poseía un lecho de plumas de gallinas. La camas de la cámara de cría observadas fueron construidas con flores secas de Trichocereus, las que son pubescentes, y una gran cantidad de ootecas de arañas (Fig. 4).

Solo muy recientemente se conoció la presencia de la garrapata blanda Argas neghmei Kohls \& Hoogstraal, 1961 (Acari: Argasidae) en los nidos de A. dorbignyi, esta ave fue su primer hospedador nativo conocido (Di Iorio et al., 2010). La restante fauna de artrópodos hallada en sus nidos, desconocida hasta el momento, constituye el objetivo de la presente contribución.

\section{Materiales y Métodos}

\section{Áreas de estudio}

Un área de estudio comprendió Campo Negro, 7 km al sur de La Poma (3050 m.s.n.m.) en la provincia de Salta (Fig. 1). A nivel florístico convergen en este lugar 3 provincias fitogeográficas bien marcadas: la vegetación del valle correspondiente al monte, dominada por especies de Larrea (Zygophyllaceae), y con cardones Trichocereus atacamensis (Phil.) Backeb. (Cactaceae); la vegetación de las laderas y cimas montañosas circundantes corresponde a las provincias de la prepuna y puna, respectivamente (Morello, 1958). La segunda área de estudio correspondió a la Cuesta de los Cardones (2900 m.s.n.m.), en la bajada inmediatamente al norte de El Infiernillo (3000 m.s.n.m.) en la provincia de Tucumán (Fig. 1), también con la misma especie de cardón. En ambas localidades los nidos de A. dorbignyi se ubicaron solamente sobre los cardones T. atacamensis (Figs. 2-3). En Campo Negro se colectaron 16 nidos durante junio de 2007: 20-VI-07, nidos 1-10; 21-VI-07, nidos 11-14; 22-VI-07, nidos 15-16,

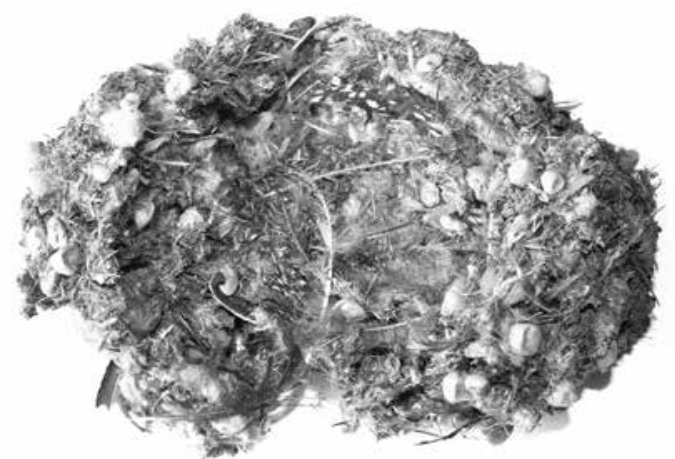

Figura 4. Cama de cría de Asthenes dorbignyi construida con flores secas de cardón y ootecas de arañas.

mientras que en la Cuesta de los Cardones, 3-VI04, se obtuvieron 4 nidos (Tabla 1).

La metodología empleada fue la de disección del hábitat descrita por Turienzo \& Di Iorio (2008). El material entomológico se encuentra depositado en la colección DIOC (Osvaldo Di Iorio, Buenos Aires, Argentina). Los especímenes de insectos muertos se indican entre corchetes [] (Tabla 1).

\section{Resultados y Discusión}

\section{Insectos en nidos de $A$. dorbignyi}

Se obtuvieron 219 y 139 insectos adultos en Campo Negro y Cuesta de los Cardones, respectivamente (Tabla 1 ), pertenecientes a 5 órdenes y 10 familias: COLEOPTERA: Carabidae [Dromius rugulosus Mateu, 1973]; Chrysomelidae: Bruchinae [Especie indeterminada]; Chysomelinae [Calligrapha sp.]; Coccinellidae [Cycloneda ancoralis (Germar, 1824); Cycloneda sicardi (Bréthes, 1925)]; Dermestidae [Trogoderma sp.]; HEMIPTERA: Coreidae [Leptoglossus crassicornis (Dallas, 1852)]; LEPIDOPTERA: Arctiidae [Especie indeterminada (pupa)]; Pyralidae [Especie indeterminada]; HYMENOPTERA: Chalcidoidea [Especie indeterminada]; Ichneumonidae [Especie indeterminada]; SIPHONAPTERA: Ceratophyllidae [Dasypsyllus sp.].

Solo se comparten 3 familias entre ambas localidades estudiadas para el invierno: Coccinellidae, Chrysomelidae y Coreidae (Tabla 1). La familia Coccinellidae (Coleoptera) fue la más abundante en ambas localidades, con $88 \%$ (Cuesta de los 
Tabla 1. Insectos y un Argasidae (Acari) encontrados en nidos de Asthenes dorbignyi de Argentina

Los ejemplares muertos dentro de los nidos se indican entre corchetes.

Salta: Campo Negro

\begin{tabular}{lllllllllllllllll}
\hline Nidos & 1 & 2 & 3 & 4 & 5 & 6 & 7 & 8 & 9 & 10 & 11 & $12-13$ & 14 & 15 & 16 & Total \\
\hline
\end{tabular}

\section{INSECTA}

\section{COLEOPTERA}

Chrysomelidae Bruchinae

Especie indeterminada

Coccinellidae

Cycloneda sicardi

$$
-\quad-\quad-\quad-\quad-\quad-\quad-11-\quad-\quad-148[33]-18[3] \quad-177[36]
$$

Dermestidae

Trogoderma sp. n.

Larvas

Adultos criados

\section{HEMIPTERA}

Coreidae

Leptoglossus crassicornis LEPIDOPTERA

Arctiidae

Especie indet. [pupa]

HYMENOPTERA

Chalcidoidea

Especie indeterminada

Ichneumonidae

Especie indeterminada

\section{ARACHNIDA}

Acari

Argasidae

Argas neghmei

\begin{tabular}{|c|c|c|c|c|c|}
\hline Nidos & 1 & 2 & 3 & 4 & Total \\
\hline \multicolumn{6}{|l|}{ COLEOPTERA } \\
\hline \multicolumn{6}{|l|}{ Carabidae } \\
\hline Dromius rugulosus & - & - & - & 2 & 2 \\
\hline \multicolumn{6}{|l|}{ Chrysomelidae Chysomelinae } \\
\hline Calligrapha sp. & - & 1 & - & - & 1 \\
\hline \multicolumn{6}{|l|}{ Coccinellidae } \\
\hline Cycloneda ancoralis & 1 & - & - & 1 & 2 \\
\hline Cycloneda sicardi & 53 & $41[1]$ & 8 & 18 & 120 \\
\hline \multicolumn{6}{|l|}{ LEPIDOPTERA } \\
\hline \multicolumn{6}{|l|}{ Pyralidae } \\
\hline Especie indeterminada & - & - & - & 1 & 1 \\
\hline \multicolumn{6}{|l|}{ SIPHONAPTERA } \\
\hline \multicolumn{6}{|l|}{ Ceratophyllidae } \\
\hline Dasypsyllus sp. & - & - & 11 & - & 11 \\
\hline \multicolumn{6}{|l|}{ HEMIPTERA } \\
\hline \multicolumn{6}{|l|}{ Coreidae } \\
\hline Leptoglossus crassic ornis & - & 1 & 1 & - & 2 \\
\hline Total por nido & 54 & $43[1]$ & 20 & 22 & 139 \\
\hline
\end{tabular}


Cardones) y 80\% (Campo Negro), siguiendo en orden de abundancia las Coreidae (Hemiptera) con $15 \%$ en Campo Negro y Ceratophyllidae (Siphonaptera) con 8\% en Cuesta de los Cardones (Figs. 5-6).

De acuerdo con la experiencia previa con los insectos en los nidos de otras aves de Argentina (Turienzo, 2012; Tutienzo \& Di Iorio 2008, 2010, 2011), la entomofauna presente en los nidos de aves puede dividirse, según los tiempos de permanencia dentro de los nidos, en residentes temporales, permanentes y accidentales u ocasionales.

\section{Residentes temporales (hibernantes)}

Coleoptera: Chrysomelidae: Bruchinae [Especie indeterminada]; Chysomelinae [Calligrapha sp.]; Coccinellidae [Cycloneda ancoralis; Cycloneda sicardi]; Hemiptera: Coreidae [Leptoglossus crassicornis].

Cycloneda sicardi fue considerada como una especie rara en revisiones taxonómicas, distribuida en Bolivia: Chuquisaca; Argentina: Salta: Dto. Cafayate, Yacochuya; Cachi; Río las Conchas, 35 km Cafayate; Tacil, 2,400 m; Catamarca: Rincón;

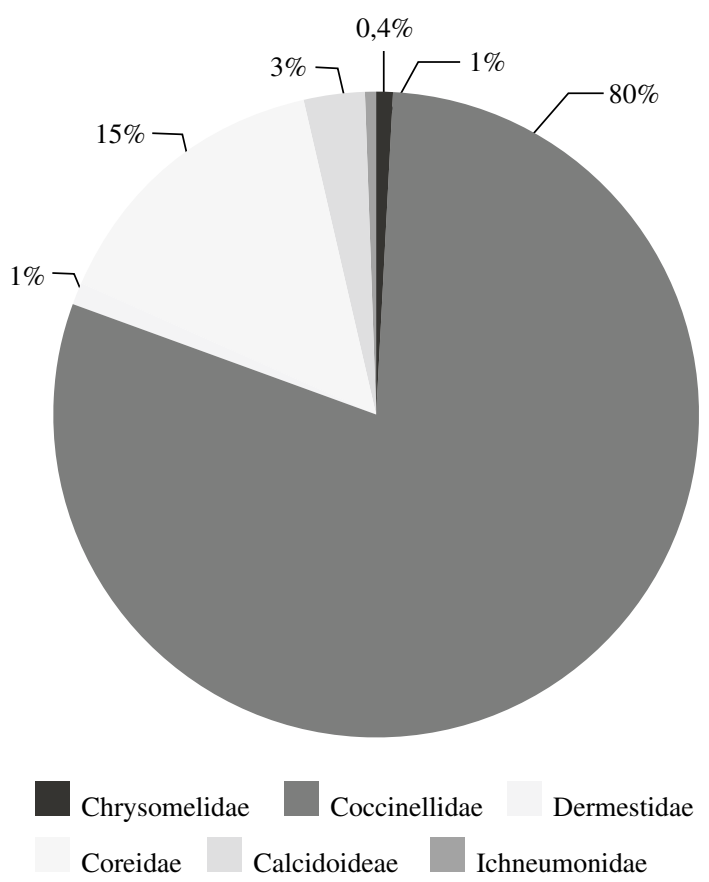

Figura 5. Composición porcentual de la entomofauna en nidos de Asthenes dorbignyi provenientes de Campo Negro, provincia de Salta, Argentina.
Camino Andalgalá-Cafayate, km 74, 2900 m; Tucumán: Zapallar; San Juan: San Juan de Cuyo; Neuquén: San Martín de los Andes (González \& Vandenberg, 2006). Se encontró un número importante hibernando en Campo Negro y en Cuesta de los Cardones, en esta última localidad junto con Cycloneda ancoralis (Tabla 1), es el primer registro de $C$. sicardi en nidos de ave. A su vez, C. sicardi es nuevo registro para las localidades mencionadas, donde aparentemente es la especie preponderante en alturas superiores a los $2500 \mathrm{~m}$. Inversamente, $C$. ancoralis es muy abundante en alturas menores (Turienzo, 2012; Turienzo \& Di Iorio, 2008, 2010, 2011).

Leptoglossus crassicornis fue registrada alimentándose sobre especies de Cactaceae del género Harrisia (1 sp.), Opuntia (7 spp.) y Tephrocactus (1 sp.) (Di Iorio, 2004), de las cuales algunas especies de los dos últimos géneros mencionados se hallan presentes en Campo Negro (Albanesi et al., 2009). También fue comúnmente observada comiendo sobre los frutos maduros de Opuntia sulphurifera en La Rioja (Di Iorio, obs. pers.). Su presencia en los nidos de A. dorbignyi se debió a adultos hibernando,

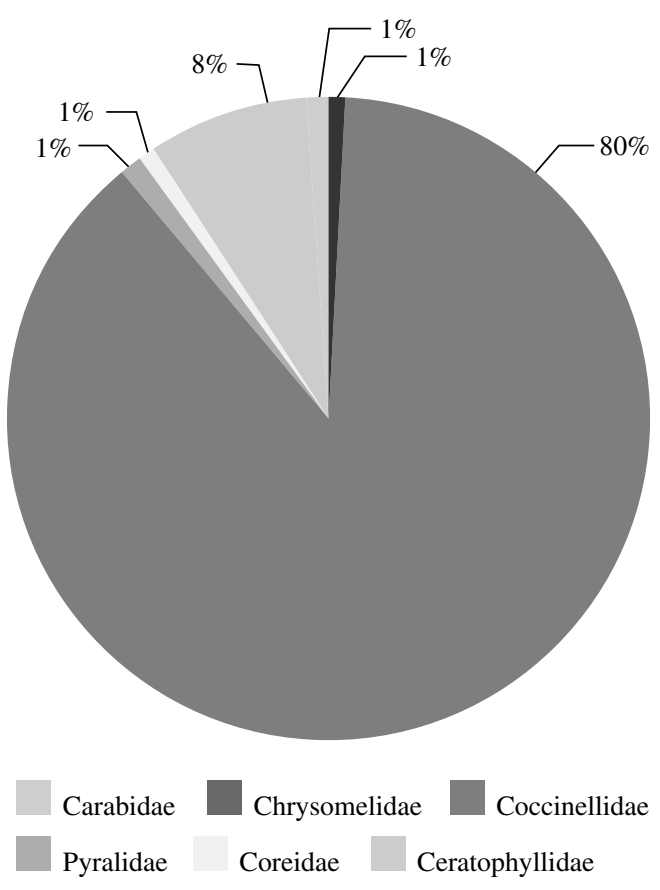

Figura 6. Composición porcentual de la entomofauna en nidos de Asthenes dorbignyi de Cuesta de los Cardones, provincia de Tucumán, Argentina. 
algo que parece ocurrir todos los años en el mismo lugar por la presencia de adultos muertos muy viejos dentro de los nidos (Tabla 1).

\section{Residentes permanentes (especies nidícolas)}

Coleoptera: Carabidae [Dromius rugulosus]; Dermestidae [Trogoderma sp.]; Siphonaptera: Ceratophyllidae [Dasypsyllus sp.].

Dromius rugulosus es conocido de Argentina: Catamarca (Sumalao); La Rioja (Patquía: Guayapa, localidad-tipo); Santiago del Estero (Choya; Fortín) y de Chile: Los Maitenes, cordillera de Santiago, 1200-1400 m (Mateu, 1973). De acuerdo con este autor, "ambas poblaciones de rugulosus no parecen demostrar una orofilia muy neta hacia las grandes alturas de la cordillera, ya que el ejemplar de Chile ha sido capturado entre 1200 y 1.400 metros de altitud y las pocas localidades argentinas por su lado no son tampoco de altura". El hallazgo de D. rugulosus en Cuesta de los Cardones (2900 m.s.n.m.) extiende considerablemente su distribución en altitud, además de ser Tucumán un nuevo registro para esta especie.

La especie de Dermestidae fue colectada como larva y criada hasta la obtención de los adultos, resultando una especie del género Trogoderma. No se corresponde con ninguna de las otras especies de Trogoderma halladas en nidos de otras aves de Argentina (Turienzo \& Di Iorio, 2008, 2010, 2011), por lo que parecería ser una especie propia de altura y muy probablemente nueva para la ciencia.

Dasypsyllus (Siphonaptera: Ceratophyllidae) es un género de pulgas que parasitan mayormente aves (Di Iorio \& Turienzo, 2009, 2011), y existen registros esporádicos sobre roedores (Hastriter \& Schlatter, 2006). Las especies de este género se distribuyen desde los Estados Unidos (Alaska), incluyendo México, Panamá, Venezuela, Perú hasta Argentina, Chile y las islas Falklands (Tabla 2). Esta amplia distribución, sin embargo, se encuentra limitada en la parte continental a la costa oeste en ambos hemisferios, estando ligada a ambientes xerófilos y de altura. Hasta el momento se han registrado a estas pulgas tanto en las aves adultas como en los nidos de 29 especies/subespecies de aves de 16 familias, incluyendo cuatro especies de Furnariidae del sur de Chile y Argentina (Tabla 2). A. dorbignyi constituye un nuevo registro de ave hospedadora para las especies del género Dasypsyllus (Tabla 2).

\section{Accidentales y/u ocasionales}

Lepidoptera: Arctiidae [Especie indeterminada (pupa)]; Pyralidae [Especie indeterminada]; Hymenoptera: Chalcidoidea [Especie indeterminada]; Ichneumonidae [Especie indeterminada].

Los Lepidoptera solo utilizan a los nidos como lugar de pupación, junto a una especie de avispa parásita (Ichneumonidae). Una avispa Chalcidoidea fue otra de las especies accidentales, probablemente parasitando las ootecas de arañas que $A$. dorbignyi utiliza en la cama de sus nidos.

\section{Vertebrados inquilinos de los nidos de Asthenes dorbignyi}

Tanto en algunos nidos de Campo Negro como de Cuesta de los Cardones se encontraron excrementos de roedores, indicando que estos nidos son también utilizados por otros animales luego de A. dorbignyi. Tres neonatos de un roedor no identificado fueron colectados en el nido \# 4 de Cuesta de los Cardones. Sin embargo, los ejemplares de Dasypsyllus fueron colectados en otro nido (Tabla 1), deshabitado en ese momento.

\section{Conclusiones}

Comparados con la diversidad y abundancia de insectos hallados en nidos de otras aves de Argentina (Turienzo, 2012; Turienzo \& Di Iorio, 2008, 2010, 2011), y situados a menos de $1000 \mathrm{~m}$ sobre el nivel del mar, los nidos de A. dorbignyi presentan una diversidad y abundancia mucho menores en su entomofauna asociada. Esta baja abundancia y riqueza de los insectos presentes (Tabla 1) se debe a las condiciones climáticas rigurosas como las existentes en los biomas de altura, lo que también fuera observado en el lado chileno de la cordillera de los Andes (Noodt et al., 1962). Sin embargo, los nidos de A. dorbignyi representan un importante refugio para una parte de la entomofauna de altura durante las estaciones más desfavorables del año.

\section{Agradecimientos}

A Armando C. Cicchino por la identifiación de la especie de Carabidae. 
Tabla 2. Especies del género Dasypsyllus (Siphonaptera: Ceratophyllidae), sus aves hospedadoras y lugares en que fueron halladas. Datos extraídos de Di Iorio \& Turienzo (2009, 2011).

\begin{tabular}{|c|c|c|c|c|}
\hline Especies & Fam. & Hospedador & Lugar & País \\
\hline Dasypsyllus (Avesopsylla) lasius lasius & HIR & Pygochelidon cyanoleuca cyanoleuca & A & ARG (BA) \\
\hline Dasypsyllus (Avesopsylla) lasius lasius & HIR & Tachycineta meyeni & $\mathrm{N}$ & ARG (TF) \\
\hline Dasypsyllus (Avesopsylla) lasius venezuelensis & HIR & Pygochelidon cyanoleuca & A-N & PAN \\
\hline Dasypsyllus (Avesopsylla) lasius venezuelensis & HIR & Especie indeterminada & $\mathrm{N}$ & VEN \\
\hline Dasypsyllus (Avesopsylla) lasius venezuelensis & IND & Especie indeterminada & $\mathrm{N}$ & PAN \\
\hline Dasypsyllus (Avesopsylla) lasius venezuelensis & IND & Especie indeterminada & $\mathrm{N}$ & PAN \\
\hline Dasypsyllus (Dasypsyllus) gallinulae & FRI & Atlapetes pallidinucha & A & $\mathrm{COL}$ \\
\hline Dasypsyllus (Dasypsyllus) gallinulae & FRI & Atlapetes schistaceus & A & $\mathrm{COL}$ \\
\hline Dasypsyllus (Dasypsyllus) gallinulae & ICT & Psarocolius viridis & $\mathrm{N}$ & VEN \\
\hline Dasypsyllus (Dasypsyllus) gallinulae & ICT & Psarocolius viridis & $\mathrm{N}$ & VEN \\
\hline Dasypsyllus (Dasypsyllus) gallinulae & THA & Grallaria flavotincta & A & $\mathrm{COL}$ \\
\hline Dasypsyllus (Dasypsyllus) gallinulae & THR & Euphonia xanthogaster & A & $\mathrm{COL}$ \\
\hline Dasypsyllus (Dasypsyllus) gallinulae & IND & Especie indeterminada & $\mathrm{N}$ & ARG (Tu) \\
\hline Dasypsyllus (Dasypsyllus) gallinulae perpinnatus & EMB & Zonotrichia capensis costaricensis & $\mathrm{N}$ & PAN \\
\hline Dasypsyllus (Dasypsyllus) gallinulae perpinnatus & PAR & Setophaga ruticilla & A & USA (Cal) \\
\hline Dasypsyllus (Dasypsyllus) gallinulae perpinnatus & TYR & Salpinctes obsoletus & $\mathrm{N}$ & USA (Cal) \\
\hline Dasypsyllus (Dasypsyllus) gallinulae perpinnatus & IND & Especie indeterminada & $\mathrm{N}$ & MEX \\
\hline Dasypsyllus (Dasypsyllus) gallinulae perpinnatus & VIR & Vireo leucophrys chiriquensis & $\mathrm{N}$ & PAN \\
\hline Dasypsyllus (Dasypsyllus) gallinulae perpinnatus & VIR & Vireo leucophrys chiriquensis & $\mathrm{N}$ & PAN \\
\hline Dasypsyllus (Neormipsyllus) aedon & FUR & Aphrastura spinicauda spinicauda & $\mathrm{N}$ & $\mathrm{CHI}$ \\
\hline Dasypsyllus (Neornipsyllus) aedon & TRO & Troglodytes aedon & $\mathrm{N}$ & $\mathrm{CHI}$ \\
\hline Dasypsyllus (Neornipsyllus) araucanus & FUR & Asthenes sp. & $\mathrm{N}$ & $\mathrm{ARG}(\mathrm{RN})$ \\
\hline Dasypsyllus (Neornipsyllus) araucanus & TYR & Xolmis pyrope & A & $\mathrm{CHI}$ \\
\hline Dasypsyllus (Neornipsyllus) comatus & $\mathrm{CHA}$ & Vanellus chilensis & A & ARG (TF) \\
\hline Dasypsyllus (Neornipsyllus) comatus & FUR & Asthenes anthoides anthoides & $\mathrm{N} ?$ & ARG (TF) \\
\hline Dasypsyllus (Neornipsyllus) comatus & FUR & Cinclodes antarcticus antarcticus & $?$ & $\mathrm{CHI}$; IF \\
\hline Dasypsyllus (Neornipsyllus) comatus & FUR & Cinclodes fuscus & $\mathrm{N}$ & $\mathrm{CHI}$ \\
\hline Dasypsyllus (Neornipsyllus) cteniopus & FUR & Aphrastura spinicauda spinicauda & $\mathrm{N}$ & $\mathrm{CHI}$ \\
\hline Dasypsyllus (Neornipsyllus) cteniopus & RHI & Scelorchilus rubecula rubecula & $\mathrm{N}$ & $\mathrm{CHI}$ \\
\hline Dasypsyllus (Neornipsyllus) cteniopus & RHI & Scytalopus magellanicus magellanicus & A & $\mathrm{CHI}$ \\
\hline Dasypsyllus (Neornipsyllus) cteniopus & TRO & Troglodytes aedon & $\mathrm{N}$ & $\mathrm{CHI}$ \\
\hline Dasypsyllus (Neornipsyllus) huinayensis & RHI & Pteroptochos tarnii & A & $\mathrm{CHI}$ \\
\hline Dasypsyllus (Neornipsyllus) huinayensis & RHI & Scelorchilus rubecula rubecula & A & $\mathrm{CHI}$ \\
\hline Dasypsyllus (Neornipsyllus) stejnegeri & $\mathrm{CHA}$ & Vanellus chilensis & A & ARG (TF) \\
\hline Dasypsyllus (Neornipsyllus) stejnegeri & FUR & Asthenes anthoides anthoides & $?$ & ARG (TF) \\
\hline Dasypsyllus (Neornipsyllus) stejnegeri & PAS & Passer domesticus & A & IF \\
\hline Dasypsyllus (Neornipsyllus) stejnegeri & TUR & Turdus falklandii falklandii & A & IF \\
\hline Dasypsyllus (Neornipsyllus) stejnegeri & IND & Especie indeterminada & $\mathrm{N}$ & MEX \\
\hline Dasypsyllus (Neornipsyllus) stejnegeri & IND & Especie indeterminada & $\mathrm{N}$ & ARG (SC) \\
\hline Dasypsyllus (Neornipsyllus) stejnegeri & EMB & Zonotrichia leucophrys & $\mathrm{N}$ & USA (Ala) \\
\hline Dasypsyllus (Neornipsyllus) stejnegeri & FRI & Phrygilus gayi & A & $\mathrm{CHI}$ \\
\hline Dasypsyllus (Neornipsyllus) stejnegeri & FUR & Aphrastura spinicauda spinicauda & $\mathrm{N}$ & $\mathrm{CHI}$ \\
\hline Dasypsyllus (Neornipsyllus) stejnegeri & ICT & Sturnella loyca & B ? & IF \\
\hline Dasypsyllus (Neornipsyllus) stejnegeri & PIC & Colaptes rupicola & $\mathrm{N}$ & PER \\
\hline Dasypsyllus (Neornipsyllus) stejnegeri & PIC & Colaptes rupicola & $\mathrm{N}$ & PER \\
\hline Dasypsyllus (Neornipsyllus) stejnegeri & TUR & Turdus falklandii magallanicus & $\mathrm{N}$ & $\mathrm{ARG}(\mathrm{SC})$ \\
\hline Dasypsyllus (Neornipsyllus) stejnegeri & IND & Especie indeterminada & $\mathrm{N}$ & MEX \\
\hline Dasypsyllus (Neornipsyllus) plumosissimus & EMB & Catamenia analis griseiventris & A & PER \\
\hline
\end{tabular}

A, sobre las aves; N, en los nidos. CHA, Charadridae; EMB, Emberizidae; FRI, Fringuillidae; FUR, Furnariidae; HIR, Hirundinidae; ICT, Icteridae; IND, Familia indeterminada; PAR, Parulidae; PAS, Passeridae; PIC, Picidae; RHI, Rhinocryptidae; THA, Thamnophilidae; THR, Thraupidae; TRO, Troglodytidae; TUR, Turdidae; TYR, Tyrannidae; VIR, Vireonidae. ARG, Argentina (BA, Buenos Aires; RN, Río Negro; SC, Santa Cruz; TF, Tierra del Fuego; Tu, Tucumán); COL, Colombia; IF, Islas Falklands; MEX, México; PAN, Panamá; PER, Perú; USA, United States of America (Ala, Alaska; Cal, California); VEN, Venezuela. 


\section{Literatura Citada}

Albanesi, N.; Magritti, L.; Pérez Michielli, M.C.

2009. Materiales y estructuras de los nidos del canastero rojizo, Asthenes dorbignyi (Reichenbach, 1853) (Aves: Passeriformes: Furnariidae). Tesis de Profesor en Ciencias Biológicas. Instituto Superior del Profesorado "Dr. Joaquín V. González". Buenos Aires, Argentina. 30 pp.

Capllonch, P.; Moyano Wagner, E.

2010. Importancia de especies de aves en ambientes altoserranos en la provincia de Tucumán, Argentina. Biológica, 12: 48-54.

De la Peña, M.R.

2005. Reproducción de las aves argentinas (con descripción de pichones). Monografia LOLA [Literature of Latin America], (20): 1-846.

Di Iorio, O.R.

2004. Hemiptera (excepto Miridae). In: Catálogo de Insectos fitófagos de la República Argentina y sus plantas asociadas. Cordo, H.A., Logarzo, G.A., Braun, K. \& Di Iorio, O.R. (Coordinadores). Sociedad Entomológica Argentina. Buenos Aires, $734 \mathrm{p}$.

Di Iorio, O.R.; Turienzo, P.

2009. Insects found in birds' nests from the Neotropical Region (except Argentina) and immigrants in the Nearctic Region. Zootaxa, (2187): 1-144.

Di Iorio, O.R.; Turienzo, P.

2011. Insects found in birds' nests from the Neotropical Region. Addenda, corrections, and the Subantarctic Region of Argentina and adjacent countries. Zootaxa, (2950): 1-108.

Di Iorio, O.; Turienzo, P.; Nava, S.; Mastropaolo, M.; Mangold,

A.J.; González Acuña, D.; Guglielmone, A.A.

2010. Asthenes dorbignyi (Reinchenbach) (Passeriformes: Furnariidae) host of Argas neghmei Kohls \& Hoogstraal, 1961 (Acari: Argasidae). Experimental and Applied Acarology, 51 (4): 419-422.

Dorst, J.

1957. The puya stands of the Peruvian high plateau as a bird habitat. Ibis, 99: 594-599.

González, G.; Vandenberg, N.J.

2006. Review of lady beetles in the Cycloneda germainii species complex (Coleoptera; Coccinellidae: Coccinellinae: Coccinellini) with descriptions of new and unusual species from Chile and surrounding countries. Zootaxa, 1311: 13-50.

Hastriter, M.W.; Schlatter, R.P.

2006. Revision of the fleas in the subgenus Dasypsyllus (Neornipsyllus) (Siphonaptera: Ceratophyllidae). Annals of Carnegie Museum, 75 (4): 247-257.

InfoNatura.

2004. Birds, mammals, and amphibians of Latin America [Web application, Version 4.1]. Arlington, Virginia: Nature
Serve. Available: http://www.natureserve.org /infonatura (acceso: agosto 17 2007).

Hoy, G.

1980. Notas nidobiológicas del noroeste argentino. II. Physis, Secc. C, Buenos Aires, 39 (96): 63-66.

Mateu, J.

1973. Los Dromius Bonelli de América (Coleopt., Lebiidae). Anales de la Escuela Nacional de Ciencias Biológicas, México, 20: 89-121.

Morello, J.

1958. La provincia fitogeográfica del monte. Opera Lilloana, 2: 5-155.

Morrison, A.

1939. The birds if the Department of Huancavelica, Peru. Ibis, 81: 453-486.

Narosky, T.; Fraga, R.; De la Peña, M.

1983. Nidificación de las aves argentinas (Dendrocolaptidae y Furnariidae). Asociación Ornitológica del Plata. Buenos Aires. 98 p.

Noodt, W.; Saiz, F.; Juhl de Noodt, H.

1962. Corte ecológico transversal de Chile central con consideración de los artrópodos terrestres. Investigaciones Zoológicas Chilenas, 8, 65-117.

Turienzo, $\mathrm{P}$.

2012. Insectos hallados en nidos de algunas especies de Furnariidae y Psittacidae (Aves) en la Argentina. Tesis de Doctorado en Ciencias Biológicas. Universidad de Buenos Aires, Facultad de Ciencias Exactas y Naturales, Departamento de Biodiversidad y Biología Experimental. Buenos Aires, 658 pp.

Turienzo, P.; Di Iorio, O.R.

2008. Insects found in birds' nests from Argentina: Anumbius annumbi (Vieillot, 1817) [Aves: Furnariidae]. Zootaxa, (1871): 1-55.

Turienzo, P.; Di Iorio, O.R.

2010. Insects found in birds' nests from Argentina. Furnarius rufus (Gmelin, 1788) and its inquiline birds, the true hosts of Acanthocrios furnarii (Cordero \& Vogelsang, 1928) [Hemiptera: Cimicidae]. Zootaxa, (2700): 1-112.

Turienzo, P.; Di Iorio, O.

2011. Insects found in birds' nests from Argentina. Myiopsitta monachus (Boddaert, 1873) [Aves: Psittacidae], exclusive host of Psitticimex uritui (Lent \& Abalos, 1946) (Hemiptera: Cimicidae). Zootaxa, (3053), 1-58.

Vaurie, $\mathrm{C}$.

1980. Taxonomy and geographical distribution of the Furnariidae (Aves, Passeriformes). Bulletin of the American Museum of Natural History, 166 (1): 1-357. 